\title{
Erratum to: Fractionated vs. single-fraction stereotactic radiotherapy in patients with vestibular schwannoma
}

\section{Hearing preservation and patients' self-reported outcome based on an established questionnaire}

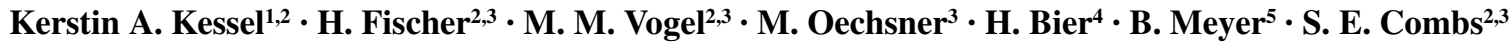

Published online: 15 December 2016

(C) Springer-Verlag Berlin Heidelberg 2016

Erratum to: Strahlenther Onkol 2016 - 192 DOI: 10.1007/ s00066-016-1070-0

Unfortunately, a typing error occurred in the results section of the abstract of the article "Fractionated vs. singlefraction stereotactic radiotherapy in patients with vestibular schwannoma".

The mean overall survival after stereotactic radiotherapy was 13.1 years and not 31.1 years. The correct sentence of the results section in the abstract reads as follows:

Mean overall survival (OS) after RT was 13.1 years, with 94 and $87 \%$ survival at 5 and 10 years, respectively.
The same applies to the German abstract. The correct sentence of the section "Ergebnisse" in the abstract reads as follows:

Das mediane Follow-up nach RT betrug 7,5 Jahre (Spanne 0-14,4 Jahre), das mittlere Gesamtüberleben (OS) nach RT 13,1 Jahre, mit Überlebensraten von 94 und 87\% nach 5 und 10 Jahren und das mittlere progressionsfreie Überleben (PFS) 13,3 Jahre, mit einem 5- und 10-JahresPFS von $92 \%$.

No further typing error occurred in the article proper.

We apologize for any inconvenience caused.

The online version of the original article can be found at http://dx. doi.org/10.1007/s00066-016-1070-0.

$\square$ Dr. Kerstin A. Kessel

Kerstin.Kessel@tum.de

1 Department of Radiation Oncology, Technical University of Munich (TUM), Ismaninger Straße 22, 1675 Munich, Germany

2 Institute of Innovative Radiotherapy (iRT), Helmholtz Zentrum München, Ingolstädter Landstraße 1, Neuherberg, Germany

3 Department of Radiation Oncology, Technical University of Munich (TUM), Ismaninger Straße 22, 81675 Munich, Germany

4 Department of Otorhinolaryngology, Technical University of Munich (TUM), Ismaninger Straße 22, Munich, Germany

5 Department of Neurosurgery, Technical University of Munich (TUM), Ismaninger Straße 22, Munich, Germany 